\title{
Nonmetallic Rigid-Flexible Outer Sheath with Pneumatic Shapelocking Mechanism and Double Curvature Structure
}

\author{
Siyang Zuo ${ }^{1}$, Ken Masamune ${ }^{1}$, Kenta Kuwana ${ }^{1}$, Morimasa Tomikawa ${ }^{2}$, Satoshi Ieiri ${ }^{2}$, \\ Takeshi Ohdaira ${ }^{3}$, Makoto Hashizume ${ }^{2,3}$, and Takeyoshi Dohi ${ }^{1}$ \\ ${ }^{1}$ Graduate School of Information Science and Technology, \\ University of Tokyo, Japan \\ ${ }^{2}$ Departments of Advanced Medicine and Innovative Technology, \\ Kyushu University Hospital, Japan \\ ${ }^{3}$ Departments of Advanced Medical Initiatives, Faculty of Medical Sciences, \\ Kyushu University, Japan \\ \{sasiyou,masa,k_kuwana\} eatre.t.u-tokyo.ac.jp, \\ \{mtomikaw, satoshi\} @med.kyushu-u.ac.jp, \\ takeshiws.ohdaira@nifty.com, mhashi@dem.med.kyushu-u.ac.jp, \\ takdohi@i.u-tokyo.ac.jp
}

\begin{abstract}
Single port access (SPA) surgery is a laparoscopic procedure using only one transumbilical-placed port. Natural orifice transluminal endoscopic surgery (NOTES) offers the possibility of surgery without visible scars. To address the access and stability problems in SPA and NOTES, we developed a device called rigid-flexible outer sheath. This sheath can be switched between flexible and rigid modes by a novel pneumatic shapelocking mechanism, and it has a double curvature structure that enables it to flex in four directions at the distal end and three directions on the rigid-flexible shaft. The insertion part of the prototype is $300 \mathrm{~mm}$ long with a $20 \mathrm{~mm}$ outer diameter, and the part is equipped with four working channels. In vivo experiments using a swine show that the outer sheath has high potential for solving access and stability problems. We expect that the outer sheath will be useful for SPA and NOTES.
\end{abstract}

Keywords: Outer sheath, Pneumatic shapelocking mechanism, SPA, NOTES, MRI-compatible.

\section{Introduction}

Single port access (SPA) surgery is an advanced, minimally invasive surgical procedure using laparoscopic devices and instruments, and it is performed through a single entry point, which is typically the patient's navel. Because the entire procedure is conducted through the navel, it does not leave any visible scar. Recent advances in minimally invasive surgery have led to the natural orifice transluminal endoscopic surgery (NOTES) technique. Because no abdominal incisions are required, a major advantage of this method is the absence of associated abdominal wall complications. While the closure of the internal entry point for NOTES presents a significant 
challenge, patients largely prefer NOTES over standard laparoscopy because of the cosmetic benefits, except if the risk is significantly higher than that in the case of the laparoscopic approach [1].

Various endoscopic devices and instruments have been researched and developed to improve surgical dexterity and to reduce specific risks and difficulties associated with SPA. However, some problems remain unsolved. Firstly, SPA requires sufficient space below the abdominal wall for surgical procedures. Secondly, while SPA is useful when the affected area is on the anterior side of the body, it is difficult to approach the affected area if it is in a deep and narrow area or on the reverse side of organs. To overcome these complications, several research groups have developed flexible manipulators. Ikuta et al. developed a micromanipulator to approach inaccessible regions [2]. Other flexible manipulators have been developed using shape memory alloys [3] or a wire-driven mechanism [4]. However, flexible instruments cannot be easily inserted into the narrow spaces between tissues or organs and cannot be completely stabilized when approaching their targets. Although current endoscopes are highly advanced, numerous problems are encountered when they are used in NOTES. During NOTES, insertion of an instrument into an area of interest without causing injury to other tissues requires a surgical instrument with a flexible mode for insertion and a rigid mode for fixing the shaft in place. Many researchers have tried to develop systems using lockable sheaths or hybrid gaits involving locking and relaxing. Robert et al. applied this idea to medical applications [5]. A type of snake robot, called HARP [6], was also developed, and the TransPort (USGI Medical, San Capistrano, CA, USA) was designed for NOTES using ShapeLock technology [7]. However, these manipulators have some limitations. Firstly, because these manipulators use wire tension to lock the shape of the shaft, they often suffer from problems of wire breakage and thus cannot be used safely. Secondly, for locking the shape of the shaft, the surgeon must control at least three wires, which is a difficult maneuver. Thirdly, the mechanisms and structures of these manipulators are complicated and costly. Finally, there is no double curvature structure and MRI compatibility is difficult to achieve.

To solve these problems associated with access and stability in SPA and NOTES, we developed a novel outer sheath. The surgeon first inserts the outer sheath through the narrow gap between the safety areas. When the outer sheath approaches the target, the surgeon locks the shape and then inserts flexible instruments easily through the path created by the sheath. This paper reports the structure of the distal end, the pneumatic shapelocking mechanism, and the structure of the wire-control operating part. In addition, the paper presents a prototype of the outer sheath. We evaluated the performance of the outer sheath through in vivo experiments using a swine.

\section{Outer Sheath Design}

\subsection{Structure of Integrated Pin-Joints at Bending Distal End}

We designed a bending distal end using an integrated pin-joint structure. Frames 1, 2, 3 , and 4 are joined together and driven by four wires that are $90^{\circ}$ apart, which facilitates flexibility in four directions (Fig. 1(A), (B)). 


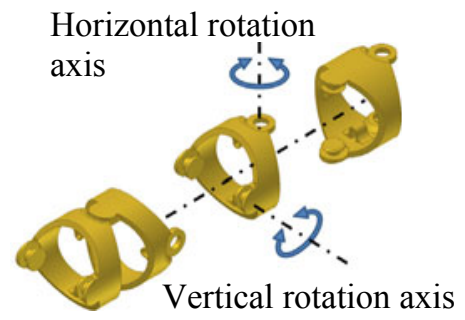

(A)

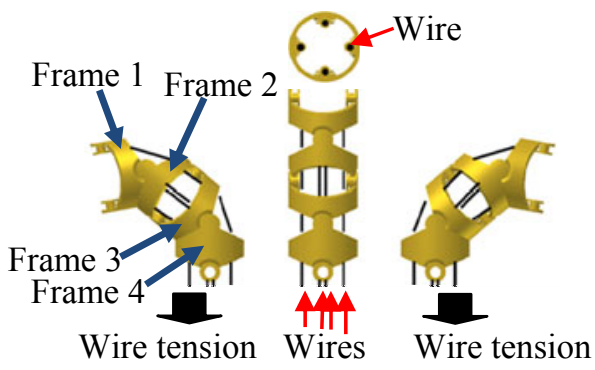

(B)

Fig. 1. Structure of bending distal end. (A) Image of units connected alternatively. (B) Mechanism of bending distal end.

The bending distal end consists of six aligned frames that mutually rotate $90^{\circ}$ around their axes $($ Fig. $4(\mathbf{C})$ ). This architecture facilitates a broad range of bending motions, resulting in ease of application during endoscopic surgery. In addition, the bending distal end is manufactured in an integrated manner, and therefore, assembly is not necessary. The integrated frame structures are produced by an Objet PolyJetbased 3D Printer (EDEN260V, Objet Geometries Ltd., Israel).

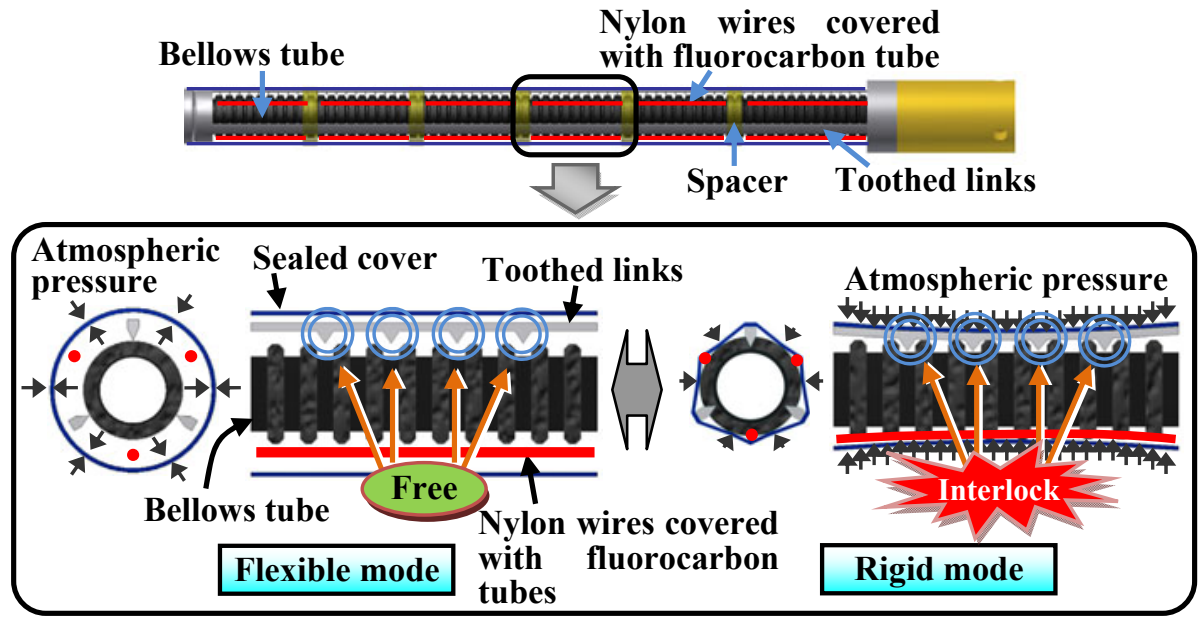

Fig. 2. Mechanism for switching between rigid and flexible modes. When there is no vacuum, the links and bellows tube can take any shape. When the pump creates a vacuum, the links move down and mesh with the ditch of the tube, and thus, the shape of the sheath is locked.

\subsection{Mechanism of Pneumatic Shapelocking on Rigid-Flexible Shaft}

With the mechanism described below, the outer sheath can alternate between flexible and rigid modes. The rigid-flexible shaft design consists of flexible toothed links, a bellows tube, a sealed cover, and nylon wires. In the flexible mode, the internal and 
atmospheric pressures equalize. Therefore, the sealed cover does not shrink, and the toothed links disengage from the bellows tube. In the rigid mode, the sealed space is evacuated by discharging the internal air, and the atmospheric pressure presses the toothed links into the bellows tube. Thus, the shape of the outer sheath is locked (Fig. 2). The bellows tube and toothed link mechanism can be easily locked as well as relaxed, providing a smooth transition between flexible and rigid modes. Furthermore, three nylon wires covered with non-shrinkable fluorocarbon tubes pass through the clearance space along the bellows tube to bend the rigid-flexible shaft of the outer sheath.

\subsection{Structure of Chain-Gear on Wire-Control Operating Part}

The wire-control operating part for the bending distal end has a chain-gear structure (Fig. 3). A surgeon can rotate the two knobs to easily control the bending angle of the distal end. Bending angles of $\pm 120^{\circ}$ in the vertical direction and $\pm 90^{\circ}$ in the horizontal direction can be achieved by Knob 1 (Fig. 3) from $-60^{\circ}$ to $+60^{\circ}$ and Knob 2 (Fig. 3) from $-70^{\circ}$ to $+70^{\circ}$, respectively. To realize MRI compatibility, the materials of the operating part are made of plastic.

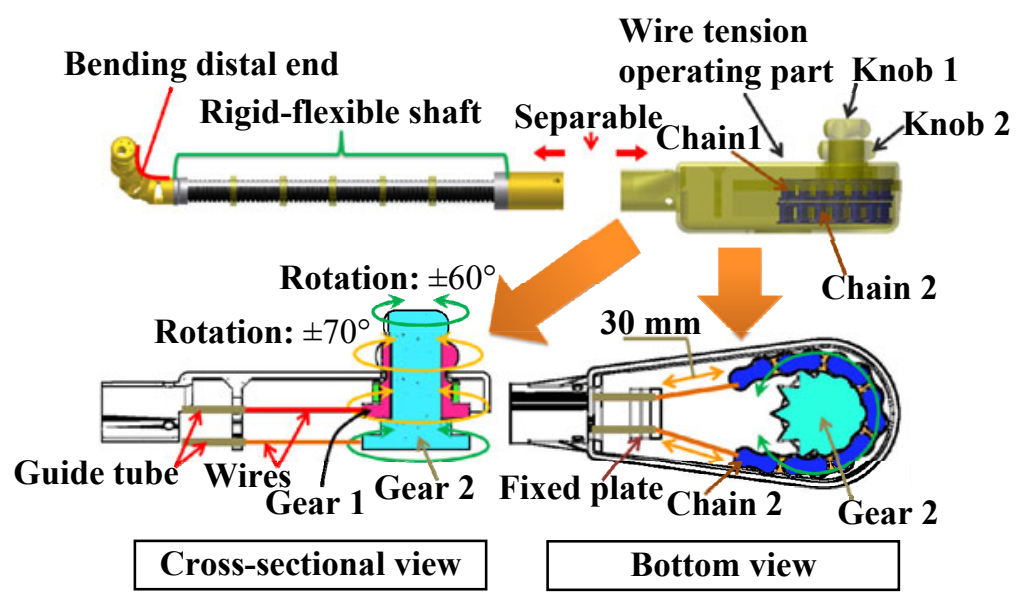

Fig. 3. Wire-control operating part for the bending distal end. Four nylon wires are connected by the chains. The bending angle of the distal end is controlled by two sets of chain-gear structures.

\subsection{Prototype}

We built a prototype of the outer sheath (Fig. 4(A), (B)). The prototype has a length of $300 \mathrm{~mm}$, maximum outer diameter of $20 \mathrm{~mm}$. The length of the bending distal end is $75 \mathrm{~mm}$. In addition, our model was equipped with one $7-\mathrm{mm}$, one $2-\mathrm{mm}$, and two $1.35-\mathrm{mm}$ working channels. The flexible instruments can be inserted from the 7-mm and 2-mm channels, and the two 1.35-mm channels are used for water jet and suction. The rigid-flexible shaft consists of three long, flexible toothed links, a bellows tube, 
and a polyethylene cover. The toothed links are $120^{\circ}$ apart, which allows the shaft to be locked in any direction. The bending distal end consists of six frames and four nylon wires. For each frame, the rotating angle in the vertical and horizontal direction is $\pm 40^{\circ}$ and $\pm 45^{\circ}$, respectively, because of which the bending distal end can achieve a curvature of $\pm 120^{\circ}$ and $\pm 90^{\circ}$ in the vertical and horizontal directions, respectively (Fig. 4(C)). The rigid-flexible shaft also has active bending capability because of three nylon wires that are $120^{\circ}$ apart, and the maximum bending angle achieved by pulling the wires is $90^{\circ}$. The wires pass through three holes in the operation part and are manually controlled. The system of the prototype is shown in Fig. 4(D). The outer sheath is separated from the vacuum controller and the vacuum source to be cleaned and sterilized. All parts of the prototype are made of plastic, and this ensures excellent MRI compatibility.

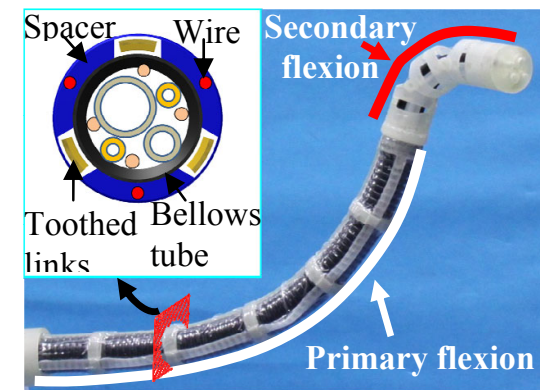

(A)

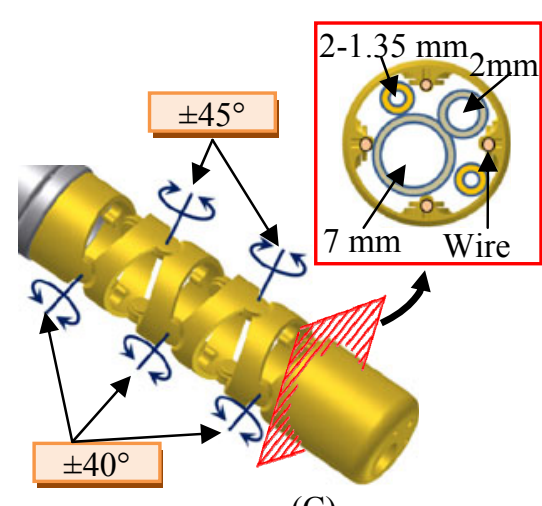

(C)

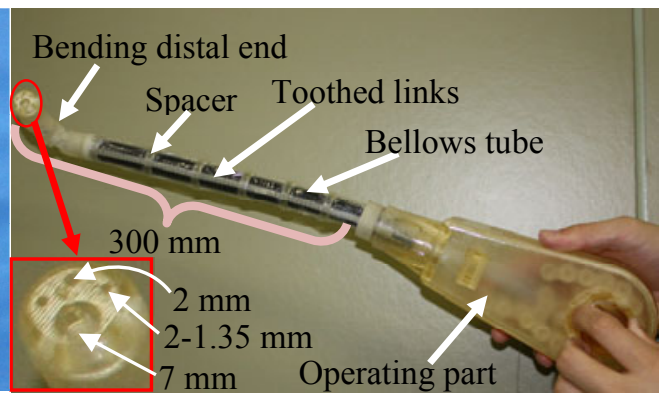

(B)

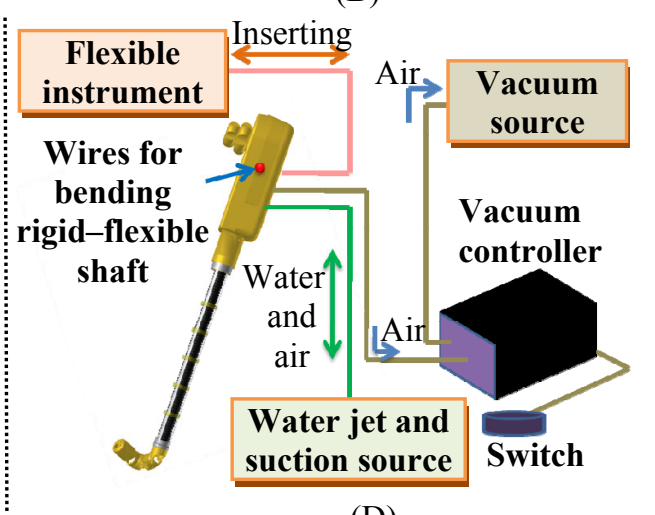

(D)

Fig. 4. Prototype of the outer sheath. (A) The outer sheath can curve like a snake and hold its shape. (B) Image of prototype. (C) Bending distal end. (D) System configuration.

\subsection{Mechanical Characteristics of Prototype}

We first examined the bending characteristics of the distal end by pulling four wires. A bending angle of $\pm 90^{\circ}$ in the horizontal direction was achieved through a wire displacement of $25 \mathrm{~mm}$ and a wire tension of $8.49 \pm 0.23 \mathrm{~N}(\mathrm{n}=3)$. A bending angle 
of $\pm 120^{\circ}$ in the vertical direction was achieved through a wire displacement of $30 \mathrm{~mm}$ and a wire tension of $11.03 \pm 0.26 \mathrm{~N}(\mathrm{n}=3)$. We next tested the bending characteristics of the rigid-flexible shaft by pulling the three wires. A maximum bending angle of $90^{\circ}$ was achieved through a wire displacement of $70 \mathrm{~mm}$ and a wire tension of $12 \pm 0.69 \mathrm{~N}(\mathrm{n}=3)$. Finally, we tested the maximum holding power of the sheath in maintaining its shape using only the pneumatic shapelocking mechanism in the rigid mode. The limited holding power was determined by visually observing the point at which deformation of the outer sheath began. The holding powers were measured in two conditions: the straight condition $\left(0^{\circ}\right)$ (Fig. 5(A)) and curved condition $\left(90^{\circ}\right)$ (Fig. 5(B)). In both cases, the load direction was the downward vertical direction. The maximum torque for which deformation of the shape did not occur was $29.55 \mathrm{~N} \bullet \mathrm{cm}$ in case (A) and $21.75 \mathrm{~N} \bullet \mathrm{cm}$ in case (B). In the flexible mode, the shape of the rigid-flexible shaft can be changed under a torque of $12 \mathrm{~N} \bullet \mathrm{cm}$ in the straight condition $\left(0^{\circ}\right)$.

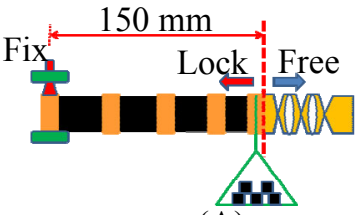

(A)

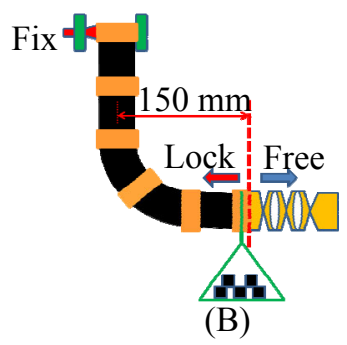

Fig. 5 Measurement of holding power in the rigid mode. (A) Load in the straight condition $\left(0^{\circ}\right)$. (B) Load in the curved condition $\left(90^{\circ}\right)$.

\section{In Vivo Experiment}

In the in vivo experiment, we tested the efficacy of our prototype in the abdominal cavity of a swine (male, $48.5 \mathrm{~kg}$ ). The surgery was performed through two access ports. We inserted a laparoscope into the port in the lower abdominal region to observe and confirm the position and shape of the outer sheath, and we inserted the outer sheath through the center of the abdominal region. The incision part during insertion of the outer sheath was protected with a LAP DISK (Hakko Medical Inc., Japan). The instruments inserted into the outer sheath were a 2.8-mm fiberscope (Sumita Optical Glass, Inc., Japan) and a 1.75-mm biopsy forceps (BF1812SF, Fujinon Toshiba ES Systems Co. Ltd., Japan). The outer sheath was connected to a vacuum pump (DTC-41, ULVAC KIKO Inc., Japan) and a vacuum controller to alternate between flexible and rigid modes by pushing a button. The sheath was also connected to a roller pump (RP-2100, Tokyo Rikakikai Co., Ltd., Japan) to jet water. Suction was applied through a vacuum supply port in the operating room.

First, we inserted the outer sheath into the gap between the liver and diaphragm to observe the posterior side of the liver with a 2.8-mm fiberscope (Fig. 6(A)). Second, we inserted the outer sheath into the abovementioned gap to locate the bladder, and we clamped the bladder surface using a biopsy forceps (Fig. 6(B)). Third, we sprayed 
water from the $1.35-\mathrm{mm}$ channel to clean the lens of the fiberscope and the internal organ, and then, we drew the water out through the second 1.35-mm channel (Fig. 6(C)). Fourth, we inserted the outer sheath into the gap among the spleen, liver, and stomach. These tasks were performed without the use of forceps (Fig. 6(D)). Finally, we tried to approach the colon from the posterior side (Fig. 6(E)). Furthermore, this prototype can be used in the rigid mode as a rigid instrument similar to conventional laparoscopic devices (Fig. 6(F)).
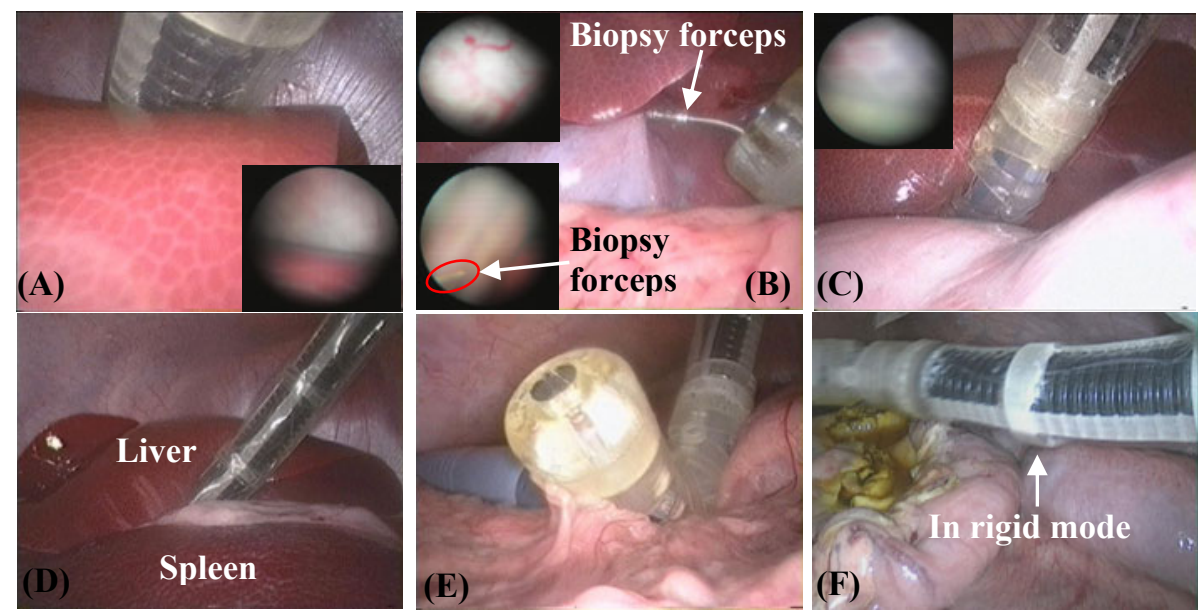

Fig. 6. Laparoscopic broad views along with fiberscope local views of the abdominal cavity of a swine. (A) Image of the outer sheath entering the gap between the liver and diaphragm. The fiberscope shows the border between the organs. (B) Image showing the clamping of liver lobes by biopsy forceps inserted through the outer sheath. The fiberscope shows the surface of the liver lobes and biopsy forceps. (C) Image showing the outer sheath during underwater observation. The fiberscope shows the underwater image. (D) The outer sheath entering the gap among the spleen, liver, and stomach. (E) The colon approached with a widely curved distal end. $(\mathrm{F})$ The prototype can perform spatial migration like rigid instruments in rigid mode.

\section{Discussion and Conclusion}

We confirmed that the bending distal end of the outer sheath enabled switching between $\pm 120^{\circ}$ and $\pm 90^{\circ}$ curvatures in the vertical and horizontal directions, respectively. The outer sheath facilitates insertion of flexible instruments into gaps between organs; therefore, even a torque of $29.55 \mathrm{~N} \bullet \mathrm{cm}$ for a straight shape and 21.75 $\mathrm{N} \bullet \mathrm{cm}$ for $90^{\circ}$ curved shapes may be sufficient against external pressure from organs and internal pressure from inserted instruments through the channels. This belief was validated through in vivo experiments. The locking mechanism guards against unintentional activation created by organs pressing against the shaft. Because the outer sheath employs vacuum as the locking mechanism at low pressures of less than $1 \mathrm{kPa}$, the tissues near the outer sheath are protected from damage even in the event of air leakage. The minimum bend radius of the rigid-flexible shaft is constrained by the 
minimum bend radius $(50 \mathrm{~mm})$ of the bellows tube. This radius corresponds to a slightly more curved path for maneuvering around small organs. However, the radius is sufficient for approach between abdominal walls and tissues or for maneuvers around large organs such as the liver.

In vivo evaluations showed that the outer sheath has strong potential for solving access problems and stability issues. The double curvature and locking capability allow the outer sheath to be positioned at the target field and to lock the shape of the rigidflexible shaft. Thus, the surgeon's hands are free. These features, combined with the endoscope's four-directional flexion, enable more complex manipulation. The outer sheath has a separate channel for water jet and suction. A 7-mm channel is designed for a 6-mm flexible endoscope. The low resolution of the 2.8- $\mathrm{mm}$ fiberscope was not adequate for observing local fields, and the 2-mm channel is not sufficiently large for standard devices such as endoscopic clips or more robust instruments. Therefore, a high-resolution scope and larger channel for robust devices are necessary for more complicated procedures. Because the locking principle of the outer sheath is based on vacuum and all parts of this device are made of plastic, the outer sheath should have little influence on MRI, which enables MRI-guided surgery [8]. Surgeons recommended that the device be used for natural orifice NOTES procedures, especially for transrectal and transvaginal NOTES, because the approach is suitable for short, gentle curves. The performance of the outer sheath with improvements in channel size and triangulation will be evaluated through future in vivo experiments on NOTES.

To secure a stable approach path for SPA and NOTES, we developed a nonmetallic rigid-flexible outer sheath that has four working channels. This sheath can flex in four directions at the bending distal end and three directions on the rigid-flexible shaft. Through in vivo experiments using a swine, we determined that flexible instruments can be inserted into deep areas in the abdominal cavity and locked into position, enabling independent flexion at the distal end. Thus, the design of the outer sheath meets the requirements for SPA and NOTES. The results of our study show that the sheath has potential for application in SPA and NOTES.

\section{References}

1. Varadarajulu, S., Tamhane, A., Drelichman, E.: Patient perception of natural orifice transluminal endoscopic surgery as a technique for cholecystectomy. J. Gastrointest. Endosc. 67, 854-860 (2008)

2. Ikuta, K., Sasaki, K., Yamamoto, K., Shimada, T.: Remote Microsurgery System for Deep and Narrow Space - Development of New Surgical Procedure and Micro-robotic Tool. In: Dohi, T., Kikinis, R. (eds.) MICCAI 2002. LNCS, vol. 2488, pp. 163-172. Springer, Heidelberg (2002)

3. Nakamura, Y., Matsui, A., Saito, T.: Shape Memory-Alloy Active Forceps for Laparoscopic Surgery. In: Proc. 1995 IEEE International Conference on Robot and Automation, vol. 3, pp. 2320-2327 (1995)

4. Simaan, N., Taylor, R., Flint, P.: High Dexterity Snake-Like Robotic Slaves for Minimally Invasive Telesurgery of the Upper Airway. In: Barillot, C., Haynor, D.R., Hellier, P. (eds.) MICCAI 2004. LNCS, vol. 3217, pp. 17-24. Springer, Heidelberg (2004) 
5. Sturges, R.H., Laowattana Jr., S.: A flexible, tendon-controlled device for endoscopy. In: Proc. 1991 IEEE International Conference on Robotics and Automation, vol. 3, pp. 25822591 (1991)

6. Amir, D., Howie, C., Alon, W., Takeyoshi, O., Marco, A.Z.: Percutaneous intrapericardial interventions using a highly articulated robotic probe. In: The First IEEE/RAS-EMBS International Conference on Biomedical Robotics and Biomechatronics, pp. 7-12 (2006)

7. Raju, G.S., Rex, D.K., Kozarek, R.A., Ahmed, I., Brining, D., Pasricha, P.J.: A novel shape-locking guide for prevention of sigmoid looping during colonoscopy. J. Gastrointest. Endosc. 59, 416-419 (2004)

8. Zuo, S., Yamanaka, N., Sato, I., Masamune, K., Liao, H., Matsumiya, K., Dohi, T.: MRICompatible Rigid and Flexible Outer Sheath Device with Pneumatic Locking Mechanism for Minimally Invasive Surgery. In: Dohi, T., Sakuma, I., Liao, H. (eds.) MIAR 2008. LNCS, vol. 5128, pp. 210-219. Springer, Heidelberg (2008) 